\title{
LAS FAMILIAS MEXICANAS CON ESTATUS INMIGRATORIO MIXTO Y LA DEPORTACIÓN MASIVA DE ESTADOS UNIDOS
}

\author{
Guillermo Yrizar Barbosa ${ }^{1}$ \\ Rafael Alarcón ${ }^{2}$
}

\begin{abstract}
Ante la creciente criminalización de la inmigración en Estados Unidos, millones de personas que forman parte de familias inmigrantes mexicanas enfrentan miedo, angustia y precariedad social. En este artículo se analiza el impacto que ha tenido la deportación masiva de mexicanos en familias con estatus inmigratorio mixto. En primer lugar, se describe la evolución de la legislación estadounidense que ha facilitado la deportación formal de extranjeros no naturalizados. Posteriormente se examinan algunas de las características sociodemográficas del flujo de personas repatriadas a México. Finalmente se presentan tres casos de padres deportados que pertenecen a familias mexicanas con estatus migratorio mixto que difícilmente pueden ser considerados como criminales.
\end{abstract}

Palabras clave: migración internacional, deportación, familias con estatus inmigratorio mixto, Estados Unidos, México.

\section{Introducción}

En este momento histórico, ante la ausencia de una reforma migratoria integral, existe una creciente criminalización de la inmigración en Estados Unidos. En consecuencia, millones de adultos, jóvenes, niñas y niños que forman parte de familias inmigrantes enfrentan miedo, angustia y precariedad social bajo el actual sistema de inmigración estadounidense. La reciente deportación masiva desde ese país representa una amenaza constante para inmigrantes mexicanos que viven en familias con estatus inmigratorio mixto.

El concepto de familia con estatus inmigratorio mixto (mixed-immigration status families), utilizado originalmente por Fix y Zimmermann ${ }^{3}$, se refiere a familias

1 City University of New York. New York, Estados Unidos.

2 El Colegio de la Frontera Norte. Tijuana, Baja California, México.

3 FIX, Michael, ZIMMERMANN, Wendy. All Under One Roof: Mixed-Status Families in an Era of Reform. 
nucleares con inmigrantes de primera generación y ciudadanos estadounidenses (generalmente los hijos) ${ }^{4}$. En otras palabras, son grupos sociales definidos por la situación o condición irregular de al menos uno de sus integrantes de acuerdo a la ley de inmigración. Estas familias también constituyen un arreglo social cada vez más común en las últimas tres décadas, cuya presencia y prevalencia reflejan la idea de Foner ${ }^{5}$ en cuanto a que la familia constituye un lugar clave para analizar las interacciones entre estructura, cultura y agencia. Si bien es cierto que durante más de cien años de historia han existido "familias migrantes", divididas por las fronteras políticas entre México y Estados Unidos o reunidas y habitando en este país - ya sean llamadas familias binacionales ${ }^{6}$ o transnacionales ${ }^{7}$, la novedad de las familias con estatus inmigratorio mixto radica en la dificultad de los migrantes indocumentados contemporáneos, especialmente mexicanos y centroamericanos, por regularizar su condición migratoria, asentarse, integrarse económica y socialmente y desarrollar proyectos familiares en Estados Unidos.

Por familias con estatus migratorio mixto nos referimos a un grupo de personas inmigrantes que están vinculadas o asociadas por parentesco o roles sociales que comparten responsabilidades en un hogar y en las que al menos uno de sus miembros no tiene la ciudadanía estadounidense y al menos algún otro miembro es ciudadano estadounidense por nacimiento o naturalización. Passel ${ }^{8}$ estimó que de un total de 6.6 millones de familias indocumentadas (o "no autorizadas") en Estados Unidos, "una parte significativa puede ser clasificada como de 'estatus mixto' - en otras palabras, familias en las que al menos uno de los padres es no-autorizado y al menos un hijo nació en Estados Unidos". Esta es la definición convencional utilizada por demógrafos y científicos sociales que se ha venido usando durante al menos los últimos 15 años.

Passel y Cohn ${ }^{9}$ argumentan que el número de niños y niñas en familias con estatus inmigratorio mixto ha crecido rápidamente en años recientes, pasando de 2.7 millones en 2003 a 4 millones en 2008. La definición y el método para calcular estos números son directos y aparentemente sin ambigüedades, sin embargo, claramente se refiere a una definición de familia que podría considerarse limitada, porque no incluye aquellos casos en que los padres $u$ otros adultos responsables de los menores en el hogar son ciudadanos y los hijos son todos indocumentados o cuando algún miembro tiene otro estatus legal para residir en Estados Unidos.

${ }_{4}$ Ver ABREGO, Leisy J., MENJÍVAR, Cecilia. Immigrant Latina Mothers as Targets of Legal Violence; DREBY, Joanna. Everyday Illegal: When Policies Undermine Immigrant Families.

5 FONER, Nancy. The Immigrant Family: Cultural Legacies and Cultural Changes.

6 CHAVEZ, Leo R. Shadowed Lives: Undocumented Immigrants in American Society.

7 MUMMERT, Gail. Pensando las familias transnacionales desde los relatos de vida: Análisis longitudinal de la convivencia intergeneracional.

8 PASSEL, Jeffrey S. The Size and Characteristics of the Unauthorized Migrant Population in the US: Estimates Based on the March 2005 Current Population Survey, p. 8.

9 PASSEL, Jeffrey S., COHN, D'Vera. A Portrait of Unauthorized Immigrants in the United States. 
El objetivo del presente artículo es analizar el impacto que ha tenido la deportación masiva de inmigrantes mexicanos en las familias con estatus inmigratorio mixto en Estados Unidos. Para este fin, en la primera parte se describe la evolución de la legislación que ha facilitado la deportación formal de extranjeros que no se han naturalizado. Posteriormente, se utilizan estadísticas descriptivas que provienen de la Encuesta sobre Migración en la Frontera Norte de México para examinar las características sociodemográficas del flujo de personas repatriadas a México por las autoridades migratorias estadounidenses. Finalmente se presentan tres casos que describen la deportación reciente de tres padres de familias mexicanas con estatus inmigratorio mixto.

\section{La legislación estadounidense y la deportación de inmigrantes}

El Departamento de Seguridad Nacional de Estados Unidos (Department of Homeland Security, DHS) distingue dos tipos de deportaciones: remociones y retornos. Las remociones (removals) son las deportaciones formales de extranjeros con base en una orden de remoción que tiene consecuencias administrativas o penales sobre reingresos subsecuentes a Estados Unidos. Por su parte, los retornos (returns) son las expulsiones de extranjeros que no se fundamentan en una orden de remoción. La mayor parte de estos retornos son de ciudadanos mexicanos que han sido aprehendidos por la Patrulla Fronteriza (Border Patrol) en el intento de cruzar la frontera ilegalmente y deportados a México ${ }^{10}$.

Después del 11 de septiembre de 2001, se empezaron a incrementar las deportaciones formales o remociones de migrantes mexicanos. El fortalecimiento del aparato legislativo para facilitar las deportaciones formales se empezó a construir en 1988 con la Anti-Drug Abuse Act de ese año (Ley contra el Abuso de Drogas), la Anti-Terrorism and Effective Death Penalty Act de 1996 (Ley sobre Antiterrorismo y Pena de Muerte Efectiva) y la Illegal Immigration Reform and Immigrant Responsibility Act de 1996 (Ley de Reforma a la Inmigración Ilegal y la Responsabilidad del Inmigrante).

De acuerdo con Doris Meissner et alii ${ }^{11}$, estas leyes han expandido el procesamiento de crímenes relacionados con inmigración así como el número de crímenes estatales y federales que conducen a la remoción, tales como crímenes de depravación moral, ofensas relacionadas con sustancias controladas, ofensas relacionadas con armas o dispositivos destructivos, crímenes de violencia doméstica o contra los niños y felonías agravadas. Asimismo, ha disminuido el poder de los jueces de inmigración para suspender la remoción de los no ciudadanos. Para FitzGerald y Alarcón ${ }^{12}$, tanto la Ley sobre Antiterrorismo y Pena de Muerte

\footnotetext{
${ }^{10}$ U.S. Department of Homeland Security (DHS). Yearbook of Immigration Statistics: 2010.

${ }^{11}$ MEISSNER, Doris et alii. Immigration Enforcement in the United States: The Rise of a Formidable Machinery, p. 92-98.

12 FITZGERALD, David, ALARCÓN, Rafael. Migration: Policies and Politics, p. 120.
} 
Efectiva como la Ley de Reforma a la Inmigración Ilegal y la Responsabilidad del Inmigrante han facilitado la deportación de extranjeros no ciudadanos que tienen que permanecer bajo detención hasta antes de ser deportados. Los inmigrantes que no son ciudadanos estadounidenses son deportados si cometen crímenes clasificados en una lista expandida de "felonías agravadas". Estos inmigrantes también están sujetos a la aplicación retroactiva de la deportación formal por crímenes por los que el castigo ya ha sido cumplido.

Las felonías agravadas, según Doris Meissner et aliii' ${ }^{13}$, es un término que sólo se usa en la ley de inmigración. En 1988, solamente había una lista de cuatro felonías, sin embargo con la promulgación de las tres leyes mencionadas anteriormente, la definición del término se expandió significativamente hasta incluir a alrededor de 50 crímenes entre los que se encuentran ofensas graves y relativamente menores, incluyendo delitos clasificados anteriormente como no graves (misdemeanors).

Desde 2003, las remociones están bajo la responsabilidad del Immigration and Customs Enforcement (ICE), que asumió la misión no exclusiva de aprehender a los indocumentados en el interior del territorio estadunidense, a diferencia de la Patrulla Fronteriza que lo hace en las fronteras. Ambas agencias pertenecen al gigantesco DHS que entró en operación en $2003^{14}$.

En 2002, el gobierno de Estados Unidos empezó a aplicar la sección 287(g) de la Ley de Reforma a la Inmigración Ilegal y la Responsabilidad del Inmigrante (IIRIRA) de $1996^{15}$ que permite al gobierno federal la posibilidad de establecer acuerdos con agencias policiales de ciudades y estados para entrenar a sus funcionarios como agentes de inmigración ${ }^{16}$.

A través del Programa de Comunidades Seguras, entre 2008 y 2014, ICE trató de identificar a los indocumentados entre las personas que purgaban condenas en las prisiones para deportarlas a sus países de origen el día de su liberación. Según Meissner et aliii ${ }^{17}$, este programa fue aplicado en prácticamente todas las 3,181 cárceles y prisiones de Estados Unidos. Este programa fue cancelado en 2014 porque no se enfocaba en los crímenes graves y en su lugar opera a partir de 2014 el Programa Prioritario de Aplicación de la Ley (Priority Enforcement Program).

El Programa Nacional de Operaciones para Fugitivos (National Fugitive Operations Program) fue establecido en 2002 para identificar y arrestar fugitivos, tales como no ciudadanos a los que se les ordenó ser removidos pero no han

\footnotetext{
${ }^{13}$ MEISSNER et alii, op. cit., p. 98.

${ }^{14}$ ALARCÓN, Rafael, BECERRA, William. ¿Criminales o Víctimas? La deportación de migrantes mexicanos de Estados Unidos a Tijuana, Baja California.

${ }^{15}$ U.S. Department of Homeland Security (DHS). The Performance of 287(g) Agreements, p. 2.

${ }^{16}$ FITZGERALD, ALARCÓN, op. cit., p. 119-120.

17 MEISSNER et alii, op. cit., p. 107.
} 
dejado el país. Conforme este programa crecía, su enfoque se desplazó de los criminales no ciudadanos a aquellos que no tenían condenas penales ${ }^{18}$.

Como consecuencia del fortalecimiento del aparato legislativo y judicial para facilitar las deportaciones formales, en los últimos años, en forma paralela al descenso en aprehensiones por parte de la Patrulla Fronteriza, se ha incrementado considerablemente la cantidad de remociones de migrantes de distintas nacionalidades. En 1999 la cifra de estos eventos apenas alcanzó los 181,072 y para 2011 la cantidad prácticamente se duplicó al alcanzar los 391,953 eventos. Como ya se ha mencionado, este proceso tiene serias consecuencias legales, incluyendo la prohibición permanente para el reingreso legal a Estados Unidos para quienes han cometido crímenes graves y hasta de 20 años para otro tipo de deportados ${ }^{19}$.

\section{Estadísticas sobre deportación y separación familiar}

La Encuesta sobre Migración en la Frontera Norte de México (EMIF Norte) proporciona información muy valiosa sobre la separación familiar de los inmigrantes mexicanos en ese país causada por las deportaciones. La EMIF Norte ha sido identificada como una de las encuestas más sobresalientes para recolectar datos sobre flujos migratorios y cruces fronterizos entre México y Estados Unidos ${ }^{20}$. Esta encuesta, administrada por el Colegio de la Frontera Norte y financiada por el gobierno mexicano, está constituida por tres grandes flujos, uno de los cuales es el de los "migrantes devueltos por las autoridades migratorias de Estados Unidos" y que define a su población objetivo como "personas de 15 años o más, nacidas en México y residentes en México o Estados Unidos devueltas por las autoridades migratorias de Estados Unidos a las autoridades migratorias de México"21. Las bases metodológicas de la EMIF Norte tienen su fundamento en técnicas que miden desplazamientos periódicos estacionales o cíclicos utilizadas en la biología. Los datos de la EMIF Norte pueden ser complementados o acompañados por otros proyectos más recientes y que permiten documentar con mayor detalle la experiencia de las personas que son expulsadas por el gobierno estadounidense, como es el caso del Estudio de Migrantes y Cruce Fronterizo (Migrant Border Crossing Study, MBCS), una encuesta con un alto nivel de validez y confiabilidad estadística ${ }^{22}$.

\footnotetext{
18 Ibidem, p. 102.

${ }^{19}$ ALARCÓN, Rafael, CALVA SÁNCHEZ, Luis. La EMIF NORTE y la deportación de migrantes mexicanos de Estados Unidos (1999-2012).

${ }^{20}$ CARRIQUIRY, Alicia, MAJMUNDAR, Malay (eds.). Estimating Illegal Entries at the U.S.-Mexico Border - Panel on Survey Options for Estimating the Flow of Unauthorized Crossings at the U.S.Mexican Border, p. 40.

${ }^{21}$ Más detalles sobre la metodología de la EMIF en: < http://www.colef.mx/emif/bases_metodologicas.php>.

22 Información sobre la metodología del MBCS en SLACK, Jeremy et alii. In Harm's Way: Family Separation, Immigration Enforcement Programs and Security on the US-Mexico Border; SLACK, Jeremy et alii. In the Shadow of the Wall: Family Separation, Immigration Enforcement, and Security.
} 
En un reporte enfocado en el flujo de los denominados "migrantes devueltos" entre 2003 y 2012 en la EMIF Norte, Velasco y Coubés ${ }^{23}$ señalan que las autoridades estadounidenses llevaron a cabo 4.6 millones de "devoluciones", es decir, eventos de expulsión de territorio estadounidense por parte de autoridades migratorias de ese país a México, sin hacer la distinción entre removidos y retornados.

En ese reporte, las autoras estiman que en 2012, un tercio de las personas devueltas a México y captadas por la EMIF Norte tenía un promedio de 8.5 años de vivir en Estados Unidos. De acuerdo con Velasco y Coubés ${ }^{24}$, "El incremento del tiempo de estadía en Estados Unidos de los deportados implica separaciones familiares y la ruptura de proyectos de vida en ese país" y añaden que debido a lo complicado que es cruzar otra vez o ir al lugar de origen "es altamente probable que las personas deportadas tengan como única opción permanecer por tiempo indefinido en las ciudades fronterizas en condiciones sumamente precarias". En el reporte se indica que el perfil del migrante "devuelto" en 2012, de acuerdo con los datos de la EMIF Norte, típicamente está conformado por varones (87 por ciento), con una edad promedio de 31 años, 8 años de educación formal, y alrededor de uno de cada cinco se refiere a Estados Unidos como el país donde reside.

Por su parte, Alarcón y Calva Sánchez ${ }^{25}$ señalan que hay una opción para analizar las características sociodemográficas de los mexicanos que son removidos de Estados Unidos utilizando los datos que ofrece la EMIF Norte a través del flujo de "devueltos". La metodología de esta encuesta no distingue entre removidos y retornados, sin embargo se puede considerar una aproximación a estas poblaciones. En este análisis se propone que los migrantes removidos sean asociados a aquellos que en la EMIF Norte indican "residir en Estados Unidos" y a los que indican "residir en México" sean asociados con los retornados.

De acuerdo con el análisis del perfil sociodemográfico de los repatriados mexicanos captados por la EMIF Norte en 1999 y 2012, se puede observar que el incremento en el tiempo de la estancia en Estados Unidos y el aumento en la edad promedio están acompañados de otros cambios en variables sociodemográficas asociadas a la configuración del núcleo familiar al que pertenece el migrante. En 1999 entre los repatriados residentes en México había una distribución equitativa entre los que eran o fueron unidos (casados, viudos y separados) y los solteros; esta situación cambió para 2012 pues la presencia de los solteros descendió a 36.3 por ciento. En cuanto a los repatriados residentes en Estados Unidos se

\footnotetext{
${ }^{23}$ VELASCO, Laura, COUBÉS, Marie Laure. Reporte sobre Dimensión, Caracterización, y Áreas de Atención a Mexicanos Deportados.

${ }^{24}$ Ibidem, p. 2.

${ }^{25}$ ALARCÓN, CALVA SÁNCHEZ, op. cit.
} 
observa la misma tendencia, en 1999 sólo el 34.5 por ciento eran solteros, pero en 2012 este porcentaje fue aún menor (20.9 por ciento).

Lo relevante no sólo es que cada vez haya más jefes de familia entre los migrantes repatriados en 2012 en comparación con 1999, sino que además un importante porcentaje de ellos cuente con familiares cercanos: hijos, hijas, cónyuge, madre o padre, en Estados Unidos, pues la deportación implica la fragmentación para estas familias. En 2012, el 71.5 por ciento de los deportados residentes en Estados Unidos indicó que al menos uno de sus familiares cercanos residía en ese país; mientras que entre los migrantes repatriados que indicaron residir en México este porcentaje fue menor (15.6 por ciento).

Se puede esperar que luego de la deportación de un migrante que forma parte de un grupo familiar que reside en Estados Unidos, la persona trate de establecerse temporalmente en México (si es que ahí cuenta con apoyo de familiares o amigos) mientras busca la manera de regresar a territorio estadounidense para reunirse con su familia. Con los datos de la EMIF Norte no se puede indagar sobre las posibles acciones futuras del grupo familiar pero sí del migrante, en particular respecto a su intención de cruzar la frontera en el futuro inmediato (dentro de los 7 días posteriores a la deportación). En 1999 la mayoría, tanto migrantes residentes en Estados Unidos (68.7\%) como residentes en México (66.8\%), indicó que intentarían "cruzar la frontera nuevamente". Para 2012, esta situación cambió drásticamente, de los residentes en Estados Unidos solamente 29 por ciento indicó que lo intentaría y 21.8 por ciento de los residentes en México $^{26}$.

El Cuadro 1 contiene estadísticas descriptivas de la EMIF Norte que permiten analizar el flujo de los mexicanos "devueltos" por las autoridades estadounidenses y que se identifican como residentes en Estados Unidos en los años de 2000 y 2012. Entre los cambios más notables en el flujo de los migrantes devueltos captados por la EMIF Norte en ambos años, encontramos que hay porcentajes más altos en 2012 de personas en matrimonios o uniones libres, jefes o jefas de familia o proveedores económicos, así como de personas que declararon tener familiares viviendo en Estados Unidos, familiares que son ciudadanos estadounidenses, incluyendo cónyuges e hijos. Es importante apuntar que el flujo de 2000 se refiere aproximadamente a 35 mil eventos de personas devueltas, mientras que en 2012 se refiere a 65 mil eventos. Estos números indican justamente el creciente número de personas que han sido aprehendidas más allá de la zona fronteriza que han pasado varios años en Estados Unidos y que se han asentado en ese país formando comunidades y familias.

${ }^{26}$ Ibidem. 
Cuadro 1 - Características sociodemográficas de los devueltos mexicanos que declararon residir en Estados Unidos en 2000 y 2012, porcentajes por género

\begin{tabular}{|l|c|c|c|c|}
\hline \multirow{2}{*}{ Variables } & \multicolumn{2}{c|}{ EMIF-N 2000 } & \multicolumn{2}{c|}{ EMIF-N 2012 } \\
\cline { 2 - 5 } & $\%$ hombres & $\%$ mujeres & $\%$ hombres & $\%$ mujeres \\
\hline Edad (media) & 28.8 & 29.1 & 35.8 & 34.6 \\
\hline Soltero & 46.4 & 33.9 & 20.7 & 21.9 \\
\hline Casado o en unión libre & 52.8 & 56.3 & 71.8 & 63.6 \\
\hline Separado o divorciado & 0.7 & 9.8 & 7.1 & 12.3 \\
\hline Jefe de familia & 66.1 & 19.3 & 81.0 & 35.2 \\
\hline Nivel educativo: menos que preparatoria & 76.7 & 75.1 & 70.1 & 68.7 \\
\hline Familiares o amigos en Estados Unidos* & 53.8 & 71.6 & 92.3 & 91.3 \\
\hline
\end{tabular}

Fuente: Elaboración propia a partir de EMIF Norte.

* Nota: La pregunta cambió. En 2000 la pregunta era específicamente acerca de la familia que vivía en la ciudad donde la persona había pasado más tiempo, mientras que en 2012 era acerca de cualquier familiar o amigos viviendo en cualquier parte de Estados Unidos.

El Cuadro 1 permite también analizar las diferencias entre hombres y mujeres en esos dos periodos. En años recientes la edad de este flujo de personas devueltas ha aumentado en aproximadamente cinco años para ambos sexos, pues en 2000 la edad media para ambos grupos no rebasa los treinta años. Además, en 2000 casi la mitad de los varones que fueron devueltos a México y declararon residir en Estados Unidos eran solteros, mientras que en 2012 solamente uno de cada cinco se encontraba en ese mismo estado civil. En 2012, el 81 por ciento de los hombres y el 35 por ciento de las mujeres que estuvieron residiendo en la Unión Americana se consideraban jefes de familia, cuando 12 años atrás los porcentajes para cada sexo eran considerablemente menores (66 y 19 por ciento respectivamente). Es también interesante notar que en años recientes el porcentaje de personas en este flujo con un nivel educativo por debajo de la preparatoria o bachillerato disminuyó, tanto para hombres como para mujeres.

\section{Criminalización de migrantes deportados: jefes de familias mixtas}

A continuación se presentan algunas narrativas reconstruidas a partir de los cuestionarios desarrollados por el equipo del Estudio de Migrantes y Cruce Fronterizo (Migrant Border Crossing Study, MBCS) con la intención de mostrar con mayor detalle el proceso de creciente criminalización, vulnerabilidad y precariedad social experimentado por inmigrantes mexicanos que viven en familias con estatus inmigratorio mixto ${ }^{27}$.

Combinando datos de 2009 a 2011, la información de la MBCS (wave II) indica que uno de cada tres de los migrantes que participaron en su encuesta realizada en ese mismo periodo, señalaron a Estados Unidos como el lugar

\footnotetext{
${ }^{27}$ Agradecemos el apoyo y las facilidades otorgadas por Dan Martínez, Jeremy Slack y el resto del equipo del MBCS para identificar y analizar estos casos.
} 
donde se encontraba su hogar actual ${ }^{28}$. El $37 \%$ de estos mismos entrevistados, es decir, de quienes identificaron su hogar actual en Estados Unidos, indicaron que intentarían realizar otro cruce dentro de la semana siguiente, comparado con $20 \%$ de los que dijeron que su hogar estaba en otro lugar. Además, "70\% de las personas que percibieron que su hogar actual se encontraba en Estados Unidos indicaron que planeaban cruzar nuevamente en el futuro [más allá de una semana], comparado con solamente $49 \%$ de aquellos que dijeron que su hogar no estaba en Estados Unidos". De acuerdo con datos de esta encuesta, entre aquellos que vivieron o trabajaron en Estados Unidos, la media de años que pasaron en ese país fue de siete.

A continuación se presentan los casos de una madre y dos padres de familias con estatus inmigratorio mixto y que fueron deportados a México recientemente. Los dos primeros casos fueron convertidos en narraciones a partir de las respuestas que dieron al equipo de la MBCS en 2011, y un caso más que no pertenece a ese estudio, está siendo documentado desde 2013.

\section{Madre de familia de Oaxaca con familia en California}

Benedicta vivió en Estados Unidos por diez años antes de ser repatriada a México en Agosto de $2011^{29}$. Ella aceptó participar en el proyecto de la MBCS un día después de que fue expulsada de Estados Unidos por autoridades migratorias. La encuesta tuvo lugar en un albergue para migrantes en Tijuana. De acuerdo con la información recolectada, Benedicta es una mujer de 38 años, casada, madre de familia, nacida en una comunidad rural oaxaqueña, y que en México no estudió más allá del sexto año de primaria. Benedicta dijo que ella quería regresar a California porque "dejó un niño del otro lado" que nació en Estados Unidos. Ella emigró de México porque su esposo estaba viviendo en la Unión Americana en esos años; cruzó la frontera en el primer intento con éxito, caminando a través de una zona remota de la frontera junto con otras 16 personas, incluyendo una mujer embarazada. Su hermano también estaba en Estados Unidos, y de hecho al momento del cruce la estaba esperando a que llegara. El coyote, que había sido contratado por un tío y financiado por la familia, les dijo que iban a caminar por cerca de 10 horas.

Pero, ¿por qué razón fue deportada Benedicta luego de pasar una década viviendo en California? De acuerdo con su historia, Benedicta estaba en la calle, recolectado latas de basura en su vecindario, cuando "vecinos racistas" Ilamaron a la policía para que la detuvieran. Ella no intentó correr antes de ser subida a la patrulla de la policía de San Diego. Tampoco dijo haber experimentado abuso verbal o físico en ningún momento. Dijo recordar y reconocer el formulario que

\footnotetext{
${ }^{28}$ SLACK et alii. In the Shadow..., op. cit., p. 15.

${ }^{29}$ Todos los nombres utilizados son seudónimos.
} 
le dieron e hicieron firmar como una "salida voluntaria". También contó que al momento de su detención y de firmar su salida voluntaria no tenía ningún documento de identificación, y de acuerdo con sus respuestas tuvo que firmar "porque no había otra opción". En no más de un día, después de haberse presentado junto con otros diez migrantes ante un juez en una corte de la zona, y luego de haber contratado a un abogado que llegó tarde al juicio, Benedicta fue regresada a México. Sobre el juicio, ella contó que no pudo entender la traducción que le proporcionaron, y también dijo no haber contado con apoyo consular. Más aún, Benedicta nunca ha tenido un pasaporte. Antes de ser deportada su mayor preocupación era que no tenía dinero y que no conocía a nadie en la ciudad fronteriza a la que fue enviada.

Benedicta vivió y trabajó en Estados Unidos por diez años, tiene hijos y hermanos que son ciudadanos de ese país. Uno de sus tres hijos era menor de edad y su esposo, que no es ciudadano estadounidense, era quien se estaba haciendo cargo de él. Benedicta no tenía ningún plan para sus hijos en caso de ser detenida y retornada a México. Ella se considera una cabeza del hogar en el que otras diez personas también habitan. También se considera una persona religiosa, católica, e incluso respondió que viviendo Estados Unidos tenía miedo de ir a la iglesia por su estatus migratorio irregular. A pesar de no hablar inglés, Benedicta es bilingüe en español y una lengua indígena hablada por al menos 50 mil personas. Incluso la considera su primera lengua. Muy probablemente pensando en su familia, cuando a Benedicta le preguntaron cuáles eran sus planes para los próximos días ella contestó que esperaba que un coyote la ayudara a cruzar. De acuerdo con algunas de las notas registradas sobre su caso, Benedicta dijo tener miedo de estar en Tijuana o de viajar a Oaxaca en autobús.

\section{Padre de familia michoacano con familia en Georgia}

Evaristo vivió en Estados Unidos tres años antes de ser repatriado en Agosto de 2011 a la ciudad de Nuevo Laredo, en el estado de Tamaulipas. Fue deportado a su país de origen cerca de las siete de la tarde, y cuando fue entrevistado dijo estar preocupado por "la situación de inseguridad" en México. Ese mismo día aceptó participar en el proyecto del MBCS, y al igual que Benedicta fue entrevistado en un albergue para migrantes. Evaristo es un hombre de 30 años de edad, que vivía en unión libre con su pareja, padre de familia, nacido en una ciudad michoacana con más de 100 mil habitantes, y dijo haber estudiado hasta el tercer año de la preparatoria o bachillerato en México. En Estados Unidos dejó un hijo menor de cinco años que es ciudadano de ese país, como lo es también su pareja con quienes vivía en un estado sureño. Antes de cruzar por primera vez la frontera los ingresos mensuales de Evaristo equivalían a 2,000 pesos mexicanos (aproximadamente 150 dólares estadounidenses en agosto del 2009). La razón por la que Evaristo salió de su lugar de origen en México fue para 
trabajar en Estados Unidos, y recuerda haber llegado a la frontera en autobús. En ese entonces cruzó por Matamoros y contando con el apoyo de familiares que residían en esa ciudad. Evaristo dijo haber cruzado con éxito en un su único intento, nadando por el río.

A Evaristo lo deportaron a México por conducir un automóvil con aliento alcohólico. Cuando la policía local lo detuvo en agosto de 2011, la única identificación que llevaba consigo era su credencial de elector mexicana. Según su testimonio estuvo un día en un centro de procesamiento donde le tomaron sus huellas, sin embargo pasó dos meses en un centro de detención esperando una resolución para su caso. Cuando se le preguntó si sabía lo que había firmado contestó inequívocamente: una deportación. A diferencia de Benedicta, a Evaristo le pareció que sí fue forzado o presionado para firmar ese formulario de deportación. Antes de que eso ocurriera reconoció haber sido tratado bien en todo momento, y al igual que Benedicta tampoco recibió ayuda consular. Evaristo explicó que fue trasladado a ver a un juez y que iba a la corte con otras personas, entre 50 y 80 migrantes, esposados y encadenados; dijo haber estado esposado por cuatro horas. Cuando le preguntaron cómo había vivido la experiencia estando en la corte su respuesta fue: "Todos estábamos muy asustados por la sentencia". Tuvo un abogado gratuito que le explicó que podía pelear su caso porque tenía familia en Estados Unidos, y quizá también por el hecho de que no había otras personas que ayudaran económicamente en su hogar, pues además él se considera el jefe del hogar que habita con su pareja y su hijo.

Finalmente el juez sentenció a Evaristo a 10 años sin poder entrar legalmente a Estados Unidos. Antes de que lo deportaran a México el único plan que este padre michoacano tenía para su hijo era que se quedara con la madre. Como Benedicta, Evaristo identifica su hogar en Estados Unidos y se considera católico, pero a diferencia del caso de la madre oaxaqueña él no se considera una persona religiosa, ni tampoco habla una lengua indígena. A pesar de no haber pasado muchos años en ese país que considera su hogar, Evaristo dijo hablar no muy bien el inglés y haber estado trabajando como mecánico. A la pregunta sobre sus planes en los siguientes días su respuesta fue esperar en la frontera a que le manden dinero para volver a cruzar a Estados Unidos, pues su destino final se encontraba en Georgia.

\section{Padre de familia mexiquense con familia en Washington ${ }^{30}$}

Era un día de mitad de semana cuando Omar, con aproximadamente 50 años de edad, fue expulsado por el gobierno estadounidense debido a su estatus migratorio irregular. Él recuerda la fecha exacta en que puso un pie en la ciudad

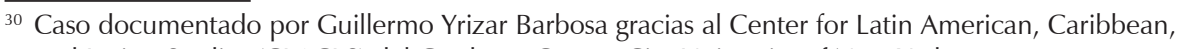
and Latino Studies (CLACLS) del Graduate Center, City University of New York. 
fronteriza del noroeste de México: un 20 de febrero. Casi una semana antes, en el día de San Valentín - o el día del amor y la amistad como se le conoce más entre los mexicanos -, alrededor de las 10 de la mañana, agentes del ICE entraron al lugar donde Omar trabajaba, una compañía pesquera en una ciudad de la costa oeste. Ahí había trabajado por casi una década.

Omar recuerda haber escuchado su nombre y asomarse a través de una pequeña ventana incrustada en la puerta de su área de trabajo, cuando vio cómo uno de sus compañeros de trabajo señalaba el lugar donde él se encontraba. Era demasiado tarde para tratar de escapar. El abogado también llegó demasiado tarde cuando Omar se encontraba en la cárcel del condado. Cuando se comenzó a documentar este caso, en agosto del 2013, Omar acababa de ser deportado y trabajaba como taxista en la ciudad de México. Sin embargo, este padre de familia se encontraba viviendo con su madre en el Estado de México, en uno de los municipios urbanos más poblados del área metropolitana del Distrito Federal. Cuando vivía en Washington Omar era un ministro religioso no católico, un pastor que se encontraba "viviendo Cristo" por al menos 18 años. Omar estaba casado con una ciudadana estadounidense de origen mexicano, y juntos tienen dos hijos que en 2013 tenían menos de 12 años y que nacieron allá. Antes de ese trágico San Valentín, Omar y su familia vivían bajo el mismo techo. En el Distrito Federal, identificándose como un "deportado", se encontraba desesperado por regresar con su familia. Su esposa también, inicialmente, estaba muy desesperada pues tenían poco dinero para mantener a los hijos. Antes de la deportación de Omar, su esposa estaba a cargo de los quehaceres domésticos, y ya con la separación tuvo que encontrar un trabajo de medio tiempo. Omar como taxista trabaja entre 10 y 12 horas al día los siete días de la semana y ya tuvo dos accidentes, uno de gravedad. "Las cosas han cambiado mucho," contaba, refiriéndose a la posibilidad de cruzar nuevamente la frontera de manera clandestina y enfrentar más riegos en comparación con el pasado. Un funcionario del gobierno estatal, luego de escuchar su caso, le dijo que su mejor opción era regresar a Estados Unidos como indocumentado.

¿Por qué fue deportado Omar? ¿Cómo fue que lo encontraron los agentes de ICE en su trabajo y lo buscaron por su nombre? Para Omar estas preguntas no tienen respuestas claras. Él reconoce que su historial migratorio durante la juventud, antes de convertirse religiosamente y formar una familia, no debió haberlo ayudado, puesto que reconoce haber cruzado clandestinamente la frontera en más de una ocasión y haber sido acusado de consumir sustancias prohibidas. También sospecha de haber cometido un error administrativo o burocrático unos años antes de su encuentro con "el hielo" (ICE). Este error consistió en contratar a un abogado para tratar de regularizar su estatus migratorio mediante su esposa e hijos nacidos en Estados Unidos, y no haberse presentado a la cita para entrevista que le fue conseguida en el consulado de ese país en 
Ciudad Juárez. Omar tampoco descarta la posibilidad de que alguna persona de su comunidad que sabía de su estatus migratorio lo hubiese denunciado ante ICE. Luego de pasar un par de años como taxista en México y haberle sido prohibido su reingreso legal a Estados Unidos por 10 años, su esposa e hijos lo han visitado en algunas ocasiones, pero para Omar, su hogar sigue estando al norte del Río Bravo.

\section{Conclusiones}

Durante las últimas tres décadas los integrantes de familias con estatus inmigratorio mixto han establecido hogares y comunidades no solo bajo la amenaza de una frontera militarizada y un sistema de deportación masiva, sino ante los crecientes obstáculos por regularizar su estatus migratorio y la posibilidad de ser identificados como criminales. En comparación con el año 2000, en años recientes la presencia de jefes de familias de migrantes mexicanos que identificaron su lugar de residencia en Estados Unidos, muchos de ellos casados o en unión libre, ha aumentado en el flujo de personas repatriadas a México ${ }^{31}$. De los 11.2 millones de personas indocumentadas en 2012, Krogstad y Passe ${ }^{32}$ estiman que 3.5 millones podrían calificar para la reciente acción ejecutiva propuesta que tiene la finalidad de diferir la deportación a los padres o madres que tienen hijos o hijas que nacieron en Estados Unidos o son residentes permanentes legales. Sin embargo, está acción ejecutiva, denominada Deferred Action for Parents of Americans and Lawful Permanent Residents (DAPA) fue bloqueada por una corte federal de Texas a principios del 2015.

Las administraciones de los presidentes Bush y Obama, se han beneficiado políticamente de la remoción masiva de inmigrantes que no se convirtieron en ciudadanos estadounidenses, bajo el argumento de que sus gobiernos están deportando formalmente a numerosos extranjeros criminales. De hecho, el presidente Obama, por las numerosas deportaciones ocurridas bajo su mandato, ha sido denominado Deporter in Chief (Deportador en Jefe). Sin embargo, es reducido el número de los removidos por delitos de alta peligrosidad como narcotráfico, asesinato, violación y secuestro, como lo ha reportado la misma Oficina del Inspector General ${ }^{33}$. Por esta razón, ha sido muy conveniente para las administraciones de los presidentes Bush y Obama, expandir la definición de criminales a todos los que han cometido delitos que no son calificados como graves $^{34}$.

\footnotetext{
31 YRIZAR BARBOSA, Guillermo. Mixed Immigration Status Families from Mexico under the US Deportation Regime: Narratives and Changes after 2000.

${ }^{32}$ KROGSTAD, Jens Manuel, PASSEL, Jeffrey S. Those from Mexico will benefit most from Obama's executive action.

${ }^{33}$ U.S. Department of Homeland Security (DHS). The Performance..., op. cit., p. 9.

${ }^{34}$ ALARCÓN, BECERRA, op. cit.
} 
Tal es el caso de la madre y los dos padres deportados cuyas historias han sido presentadas aquí. Benedicta parece haber sido deportada por haber estado recolectando latas de basura en su vecindario. Evaristo fue deportado por conducir un automóvil con aliento alcohólico, lo que se denomina conducir bajo la influencia del alcohol. Omar fue deportado por haber cruzado clandestinamente la frontera en más de una ocasión y por haber sido acusado de consumir sustancias prohibidas. Probablemente su caso se convirtió en sospechoso cuando no se presentó a la cita con las autoridades migratorias en el consulado estadounidense de Ciudad Juárez. De cualquier manera, en ninguno de estos casos, la madre y los dos padres de familia se pueden considerar como criminales.

Desde finales de la década de los ochenta el estatus migratorio indocumentado se ha venido convirtiendo en una condición social y legal indeseable que afecta negativamente la integración y el futuro de inmigrantes de primera generación y millones de ciudadanos de ese país. Cada vez que una madre o un padre de familia con estatus inmigratorio indocumentado es deportada o deportado, las posibilidades de movilidad social ascendente o de una vida familiar estable se reducen significativamente para ellos, sus cónyuges y para sus descendientes inmediatos, sean ciudadanos estadounidenses o no. Esta situación representa una gran contradicción para un "país de inmigrantes" que se considera un gran promotor de la democracia y los derechos humanos.

\section{Bibliografía}

ABREGO, Leisy J.; MENJÍVAR, Cecilia. Immigrant Latina Mothers as Targets of Legal Violence. International Journal of Sociology of the Family, v. 37, n. 1, 2011, p. 9-26. ALARCÓN, Rafael; BECERRA, William. ¿Criminales o Víctimas? La deportación de migrantes mexicanos de Estados Unidos a Tijuana, Baja California. Norteamérica, v. 7, n. 1. Enero - Junio, 2012, p. 125-148.

ALARCÓN, Rafael; CALVA SÁNCHEZ, Luis. La EMIF NORTE y la deportación de migrantes mexicanos de Estados Unidos (1999-2012). In NAVA PÉREZ, Alma Rosa; LEITE, Paula (coords.). 20 años de la Encuesta sobre Migración en la Frontera Norte de México. México: Consejo Nacional de Población, 2014.

CARRIQUIRY, Alicia; MAJMUNDAR, Malay (eds.). Estimating Illegal Entries at the U.S.Mexico Border - Panel on Survey Options for Estimating the Flow of Unauthorized Crossings at the U.S.-Mexican Border. Washington, D.C: The National Academies Press, 2013.

CHAVEZ, Leo R. Shadowed Lives: Undocumented Immigrants in American Society. Fort Worth: Cengage Learning, 1997.

DREBY, Joanna. Everyday Illegal: When Policies Undermine Immigrant Families. CA: University of California Press, 2015.

FITZGERAlD, David; ALARCÓN, Rafael. Migration: Policies and Politics. In SMITH, Peter H.; SELEE, Andrew (eds.). Mexico and the United States: The Politics of 
Partnership. Boulder y Londres: Lynne Rienner Publishers, 2013.

FIX, Michael; ZIMMERMANN, Wendy. All Under One Roof: Mixed-Status Families in an Era of Reform. International Migration Review, v. 35, n. 2, 2001, p. 397-419.

FONER, Nancy. The Immigrant Family: Cultural Legacies and Cultural Changes. International Migration Review, v. 31, n. 4, 1997, p. 961-974.

KROGSTAD, Jens Manuel; PASSEL, Jeffrey S. Those from Mexico will benefit most from Obama's executive action. Washington, DC: Pew Hispanic Center, 2014. Disponible en: http:/www.pewresearch.org/fact-tank/2014/11/20/those-frommexico-will-benefit-most-from-obamas-executive-action/.

MEISSNER, Doris; KERWIN, Donald M.; CHISHTI, Muzaffar; BERGERON, Claire. Immigration Enforcement in the United States: The Rise of a Formidable Machinery. Washington, DC: Migration Policy Institute, 2013. Disponible en: <http:// www.migration policy.org/research/immigration-enforcement-united-states-riseformidable-machinery $>$.

MUMMERT, Gail. Pensando las familias transnacionales desde los relatos de vida: Análisis longitudinal de la convivencia intergeneracional. In ARIZA, Marina; VELASCO, Laura (coords.). Métodos cualitativos y su aplicación empírica: Por los caminos de la investigación sobre migración internacional. México D.F.: UNAM y COLEF, 2012.

PASSEL, Jeffrey S.; COHN, D'Vera; GONZALEZ-BARRERA, Ana. Net Migration from Mexico Falls to Zero - and Perhaps Less. Washington, DC: Pew Hispanic Center, 2012. PASSEL, Jeffrey S.; COHN, D'Vera. A Portrait of Unauthorized Immigrants in the United States. Washington DC: Pew Hispanic Center, 2009. Disponible en: < http://www. pewhispanic.org/files/2012/04/PHC-Net-Migration-from-Mexico-Falls-to-Zero.pdf>. PASSEL, Jeffrey S.; COHN, D'Vera. Trends in Unauthorized Immigration: Undocumented Inflow Now Trails Legal Inflow. Washington, DC: Pew Hispanic Center, 2008.

PASSEL, Jeffrey S. The Size and Characteristics of the Unauthorized Migrant Population in the US: Estimates Based on the March 2005 Current Population Survey. Washington DC: Pew Hispanic Center, 2006. Disponible en: <http://www.pewhispanic.org/ files/reports/61.pdf >.

SLACK, Jeremy; MARTíNEZ, Daniel; WHITEFORD, Scott; PEIFFER, Emily. In Harm's Way: Family Separation, Immigration Enforcement Programs and Security on the US-Mexico Border. Journal on Migration and Human Security, v. 3, n. 2, 2015, p. 109-128.

SLACK, Jeremy; MARTÍNEZ, Daniel; WHITEFORD, Scott; PEIFFER, Emily. In the Shadow of the Wall: Family Separation, Immigration Enforcement, and Security. CLAS, University of Arizona, 2013.

VELASCO, Laura; COUBÉS, Marie Laure. Reporte sobre Dimensión, Caracterización, y Áreas de Atención a Mexicanos Deportados. Tijuana: El Colegio de la Frontera Norte, 2013. Disponible en: <http://www.colef.mx/wp-content/uploads/2014/01/ Reporte-Deportos.pdf >.

U.S. Department of Homeland Security (DHS). Yearbook of Immigration Statistics: 2010. Washington, D.C.: U.S. Government Printing Office, 2011. 
U.S. Department of Homeland Security (DHS). Office of the Inspector General, 2010. The Performance of 287(g) Agreements. Washington, D.C.: U.S. Department of Homeland Security OIG-10-63. March 2010.

YRIZAR BARBOSA, Guillermo. Mixed Immigration Status Families from Mexico under the US Deportation Regime: Narratives and Changes after 2000. Ponencia presentada en el congreso anual la Asociación de Estudios Latinoamericanos (LASA). San Juan, Puerto Rico, 2015.

\section{Abstract \\ Mexican families with mixed immigration status and massive deportation from the United States}

Because of the growing criminalization of immigrants in the United States, millions of people who are part of Mexican immigrant families are facing fear, anguish, and social precarity. This article analyses the impact of the massive deportations of Mexicans who belong to families with mixed immigration status. First, a description is given of the evolution of U.S. legislation that has facilitated the formal deportation of non-naturalized immigrants. Then, some socio-demographic characteristics of the flow of people repatriated to Mexico are examined. Finally, three cases of deported parents, who belong to Mexican families with mixed migration status and can hardly be considered criminals, are presented.

Keywords: international migration, deportation, families with mixed immigration status, United States, Mexico.

Recibido para publicación en 16/07/2015

Aceptado para publicación en 22/10/2015

Received for publication in July, $16^{\text {th }}, 2015$

Accepted for publication in October, 22 $2^{\text {th }}, 2015$

ISSN impresso 1980-8585

ISSN eletrônico 2237-9843

http://dx.doi.org/10.1590/1980-85852503880004504 